\title{
Three-Dimensional in situ Reconstructions of Microstructures with Bimodal Grain Size Distributions
}

Ashley Bucsek ${ }^{1 *}$, Lee Casalena ${ }^{2}$, Darren C. Pagan ${ }^{3}$, Partha P. Paul ${ }^{4}$, Yuriy Chumlyakov ${ }^{5}$, Michael J. Mills $^{6}$ and Aaron P. Stebner ${ }^{7}$

1. Aerospace Engineering and Mechanics, University of Minnesota, Minneapolis, MN, USA.

2. Thermo Fisher Scientific, Hillsboro, OR, USA.

3. Cornell High Energy Synchrotron Source, Ithaca, NY, USA.

4. Mechanical Engineering, Northwestern University, Evanston, IL, USA.

5. Department of Physics of Metals, Tomsk State University, Tomsk, Russia.

6. Materials Science \& Engineering, The Ohio State University, Columbus, OH, USA.

7. Mechanical Engineering, Colorado School of Mines, Golden CO, USA.

* Corresponding author: abucsek@umn.edu

Solid-state shape memory alloy (SMA) actuators are compact, scalable, and capable of supporting and generating large mechanical loads while weighing a fraction of traditional actuators. As a result, SMAs are an enabling technology for biomedical, active damping, and aerospace actuation systems [1,2]. The vast majority of SMAs, however, do not exhibit perfectly reversible actuation performances. There is an inherent cyclic instability that causes the mechanical response of an SMA to change from cycle to cycle. This instability is known as functional fatigue and is understood to originate from defects such as dislocations and grain boundaries that are generated under actuation conditions. Recently, Bowers et al. [3] and Casalena [4] used electron microscopy to observe the emergence of low-volume grains with large, well-defined misorientations ( $\Sigma$ values) after 20-100 actuation cycles. However, the small sampling sizes required for electron microscopy prevented them from reporting how many actuation cycles were needed for the special $\Sigma$ boundaries to form, how prevalent the boundaries were, or what a representative volume is for observing these boundaries. To answer these questions, we used far-field high-energy diffraction microscopy (ff-HEDM), near-field high-energy diffraction microscopy (nfHEDM), and microcomputed tomography $(\mu \mathrm{CT})$ to evaluate the structure of emergent high-angle $\Sigma$ grain boundaries throughout the volume of a mm-scale single-crystal nickel-titanium sample under actuation conditions [5]. Three-dimensional in situ reconstructions are presented, including the structure and distribution of emergent $\Sigma$ grain boundaries (Figure 1). The low volume and uneven dispersion of the emergent $\Sigma$ boundaries across the volume show why previous electron microscopy investigations of $\Sigma$ grain boundary formation were inconsistent.

One important byproduct of the nf-HEDM grain reconstructions when studying microstructures with a large grain size disparity is that large grains tend to "dominate" small grains. When a grain is reconstructed, the completeness (or confidence) of the grain decays beyond the grain boundary, schematized in one dimension in Figure 2. For samples with a uniform grain size distribution, this has little effect; the completeness values will decay at comparable rates, and the grain boundary will be reconstructed accurately (Figure 2B). For samples with a bimodal grain size distribution, the completeness of very large grains can extend over neighboring small grains (Figure $\mathbf{2 A}$ ). In these cases, the large grains will be reconstructed, but the small grains will not. Furthermore, because small grains have low volume and thus low intensity, the reflections of the small grains are sometimes only detected on the brightest (hkl) rings, resulting in lower completeness values for the small grains than large grains and increasing the dominance of large grains over small. To capture the emergence of the new, low- 
volume regions, we reconstructed the emergent low-volume orientations separately and then added them to the final reconstruction. In other words, we identified the low-volume regions and gave them preference over the large-volume regions in the reconstruction. Thus, this study illustrates how HEDM can be used to investigate microstructures with significant grain size disparity [6].

\section{References:}

[1] O Benafan, DJ Gaydosh, Smart Mater. Struct. 26 (2017), p. 095002.

[2] T Deurig, AR Pelton, D. Stöckel, Mater. Sci. Eng. A 50 (1999), p. 15.

[3] ML Bowers et al., Acta Mater. 91 (2015), p. 318.

[4] L Casalena, The Ohio State University (2017).

[5] AN Bucsek et al., Scr. Mater. 162C (2019), p. 361.

[6] The authors gratefully acknowledge the support provided by NSF GRFP (DGE-1057607), NSF (CMMI-1454668), XSEDE (TG-MSS160032, TG-MSS170002), DOE (DE-SC0001258), and the support for CHESS provided by the NSF (DMR-1332208, DMR-0936384).

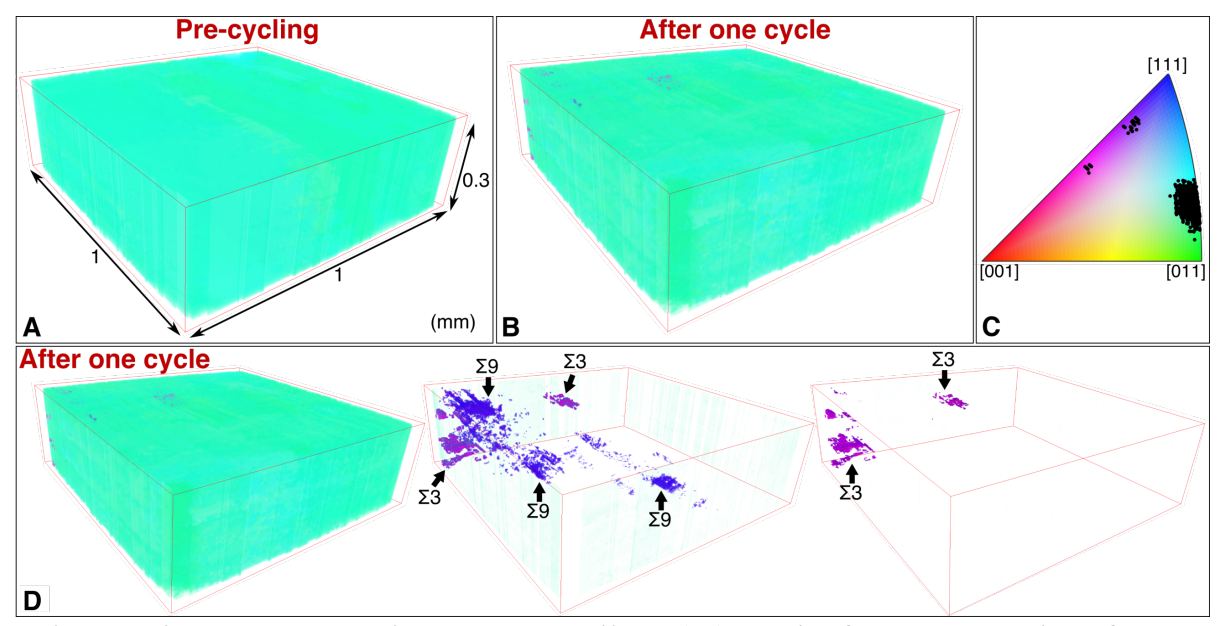

Figure 1. 3D in situ grain reconstructions pre-cycling (A) and after one cycle of actuation (B) colored by orientation $(C)$. The after one cycle grain map is shown again in (D) with varying transparency to reveal the internal structure of the new grains. Figure reprinted from [5].

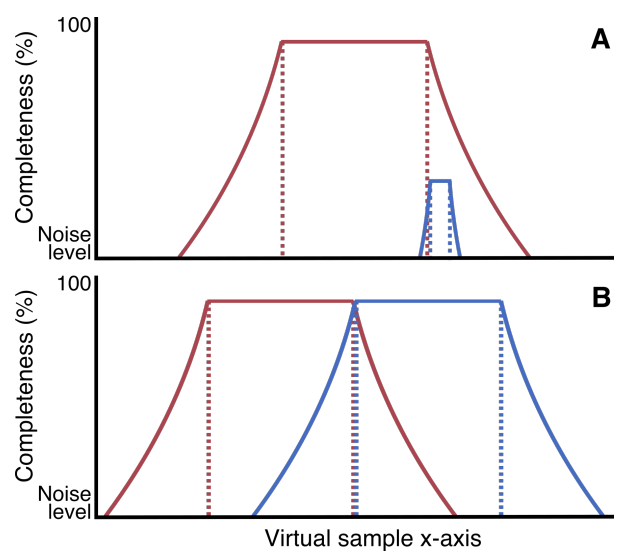

Figure 2. One-dimensional illustration showing the effect of disparate (A) and similar (B) grain sizes on reconstruction completeness levels. The dotted lines illustrate where the grain actually ends, and the solid lines show the completeness values. Figure reprinted from [5]. 